\title{
Subanesthetic ketamine for pain management in hospitalized children, adolescents, and young adults: a single-center cohort study
}

This article was published in the following Dove Press journal:

Journal of Pain Research

5 April 2017

Number of times this article has been viewed

\author{
Kathy A Sheehyl,* \\ Caroline Lippold',* \\ Amy L Rice' \\ Raissa Nobrega' \\ Julia C Finkel'
}

Zenaide MN Quezado',2

'Division of Anesthesiology, Pain, and Perioperative Medicine, The Sheikh

Zayed Institute for Pediatric Surgical Innovation, Children's Research Institute, Children's National Health System, George Washington University School of Medicine and Health Sciences, ${ }^{2}$ Center for Neuroscience Research, Children's Research Institute, Children's National Health System, Washington, DC, USA

*These authors contributed equally to this work
Correspondence: Zenaide MN Quezado Department of Perioperative Medicine National Institutes of Health Clinical Center National Institutes of Health 10 Center Drive Building 10, Room 6-5561 Bethesda, MD 20892, USA Email zquezado@nih.gov
Background: Subanesthetic doses of ketamine, an $\mathrm{N}$-methyl-D-aspartate receptor antagonist used as an adjuvant to opioid for the treatment of pain in adults with acute and chronic pain, have been shown, in some instances, to improve pain intensity and to decrease opioid intake. However, less is known about the role of ketamine in pain management in children, adolescents, and young adults.

Purpose: We examined the effects of subanesthetic ketamine on pain intensity and opioid intake in children, adolescents, and young adults with acute and chronic pain syndromes treated in an inpatient setting.

Methods: This is a longitudinal cohort study of patients treated with subanesthetic ketamine infusions in regular patient care units in a tertiary pediatric hospital. Primary outcomes included changes in pain scores and morphine-equivalent intake.

Results: The study cohort included 230 different patients who during 360 separate hospital admissions received subanesthetic ketamine infusions for pain management. Overall, ketamine infusions were associated with significant reductions in mean pain scores from baseline (mean pain scores 6.64 [95\% CI: 6.38-6.90]) to those recorded on the day after discontinuation of ketamine (mean pain scores 4.38 [95\% CI: 4.06-4.69]), $p<0.001$. Importantly, the effect of ketamine on pain scores varied according to clinical diagnosis $(p=0.011)$, infusion duration ( $p=0.004)$, and pain location $(p=0.004)$. Interestingly, greater reductions in pain scores were observed in patients with cancer pain and patients with pain associated with pancreatitis and Crohn's disease. There were no records of psychotomimetic side effects requiring therapy.

Conclusion: These data suggest that administration of subanesthetic ketamine for pain management is feasible and safe in regular inpatient care units and may benefit children, adolescents, and young adults with acute and chronic pain. This study is informative and can be helpful in determining sample and effect sizes when planning clinical trials to determine the role of subanesthetic ketamine infusions for pain management in pediatric patients.

Keywords: cancer pain, sickle cell disease, CRPS, postoperative pain, chronic pain, acute pain

\section{Introduction}

Ketamine has been used clinically since the 1960 s as a general anesthetic. ${ }^{1}$ A decade after its approval by the US Food and Drug Administration, researchers showed that ketamine reduces the excitatory response to $N$-methyl-D-aspartic acid (NMDA) in central neurons, thus revealing its NMDA receptor antagonism properties ${ }^{2}$ and advancing our understanding of the pharmacology of ketamine. Currently, clinicians use ketamine as a general anesthetic less frequently, given its emergence psychotomimetic effects and findings of neurotoxic effects shown in the developing brain of animals. ${ }^{3}$ Conversely, 
over the past decade, researchers have explored the effect of subanesthetic doses of ketamine as an antidepressant ${ }^{4}$ and as an analgesic. ${ }^{5,6}$

The use of subanesthetic ketamine as an analgesic is supported by evidence, from both animal and human studies, implicating activation of NMDA receptors in the pathobiology of nociceptive, inflammatory, and neuropathic pain and in central sensitization. ${ }^{7}$ In addition, activation of NMDA receptors has also been shown in animals ${ }^{8}$ and in human experimental studies ${ }^{9}$ to play a role in settings where acute or chronic use of opioids is associated with tolerance or opioidinduced hyperalgesia. Therefore, based on these preclinical findings, ketamine has been examined clinically as an adjuvant to opioids for the treatment of acute and chronic pain and in settings of opioid-induced hyperalgesia. ${ }^{6,10}$ However, among clinical studies, there has been a great variability in ketamine doses and duration of administration. Additionally, the beneficial effects of ketamine have not been consistently reported, and some groups have been unable to show opioidsparing effects of ketamine in clinical settings associated with opioid-induced hyperalgesia. ${ }^{11,12}$ Similarly, in chronic pain, there are no definitive data supporting the use of ketamine for the treatment of chronic regional pain syndrome (CRPS) or other types of chronic pain. ${ }^{13}$ Further, in critical reviews of available trials, researchers can only conclude that there might be a beneficial role for ketamine as an adjuvant to opioids to treat pain in the postoperative period, cancer pain, and chronic pain. ${ }^{9,12,14,15}$ Consequently, as outlined in clinical guidelines, the evidence to support the use of ketamine for the treatment of acute and chronic pain is at best moderate. ${ }^{16,17}$

However, despite lack of conclusive clinical evidence to support its use, clinicians from various disciplines in many countries continue to use ketamine as an adjuvant to opioids for acute and chronic pain management. ${ }^{18-21}$ Some argue that in the setting of limited effective pharmacologic options, one might be left with no alternative other than less studied strategies such as ketamine infusions. Our institution has used subanesthetic ketamine (defined as not $>1 \mathrm{mg} / \mathrm{kg} / \mathrm{h}$ ) to treat acute and chronic pain in children, adolescents, and young adults since 2006 . We have previously reported our experience using ketamine for the treatment of cancer pain, postoperative, and chronic pain. ${ }^{5,11}$ In this study, we sought to examine the use of subanesthetic ketamine in a large number of patients admitted to a tertiary pediatric hospital whose pain proved unresponsive to routine pharmacologic interventions. Specifically, we sought to determine the overall effect of ketamine on pain scores and opioid intake, to identify factors associated with larger or smaller effects and to examine the frequency with which ketamine was associated with clinically meaningful reductions in pain scores and opioid intake.

\section{Methods Data collection}

The Children's National Health System Institutional Review Board approved this longitudinal cohort study and a waiver for written informed consent in 2014 (Pro00004569). Requirement for patient written consent to review their records was waived as this was a minimal risk study and the data collected were devoid of patient identifiers. Patients who had received subanesthetic doses of ketamine for pain management were eligible for inclusion in this study. We reviewed a list of all patients (obtained from the hospital pharmacy records) who were admitted between January 2006 and April 2014 and had received an order for continuous infusion of intravenous ketamine. Patients who lacked a pain medicine consult, underwent a spinal fusion procedure (those patients were part of a previously reported investigational study ${ }^{11}$ ), had incomplete record of pain scores and/or opioid intake, or received ketamine only for the purpose of sedation were excluded. Data collected included sex, age, race, ketamine infusion duration, pain duration (acute vs chronic), pain diagnoses (vaso-occlusive episodes [VOE], visceral, CRPS, headache, or other [mucositis, diabetic neuropathy, neurofibromatosis, and postural orthostatic tachycardia syndrome]), primary clinical diagnosis (accidental trauma, surgical trauma, and postoperative pain [patients who had a planned surgical procedure during hospitalization], functional gastrointestinal disorder [chronic and/or recurrent gastrointestinal symptoms with no identified etiology], inflammatory disease [pancreatitis or Crohn's disease], sickle cell disease, malignancy, or other), and primary pain location. Patients with pain in more than two locations were classified as having generalized pain. From the medical charts, we retrieved documentation of hemodynamic changes that warranted treatment with vasoactive agents, as well as reports of psychotropic effects, nausea, vomiting, and changes in sleep pattern. All pain scores recorded throughout admission days were collected. We then calculated average daily pain scores before (day prior to ketamine administration) and after ( $24 \mathrm{~h}, 48 \mathrm{~h}$, and on the day after discontinuation) ketamine infusion. Pain scores were measured using the Numeric Rating Scale (NRS), the Wong-Baker FACES ${ }^{\circledR}$, or the Face, Legs, Activity, Cry, and Consolability (FLACC), as appropriate. At the times indicated earlier, we also collected total daily opioid intake and then calculated the daily 
oral morphine-equivalent (per body weight in kilograms) intake using the opioid conversion tool available at www. globalrph.com/narcoticonv.htm. ${ }^{22}$

\section{Outcomes}

The primary outcome was change in average pain scores from baseline to postketamine infusion. Secondary outcomes included changes in oral morphine-equivalent intake from baseline measurements to postketamine infusion and proportion of ketamine infusions that resulted in clinically meaningful reductions in pain scores and opioid intake. We defined clinically meaningful change in pain scores or opioid intake as a $\geq 20 \%$ reduction in pain scores and in morphine-equivalent intake from baseline to the day after ketamine discontinuation (day after ketamine - baseline/ baseline $\times 100)$. Such a decline in pain score $(20 \%)$ is larger than the $12.5 \%$ decrease in pain score suggested by others as being minimally clinically significant for adolescents with chronic pain. ${ }^{23}$

\section{Ketamine administration}

At our institution, ketamine prescribed by the Pain Medicine Division can be administered on regular inpatient care units where oxygen delivery set-up, suction, and resuscitation equipment are available. Trained nurses administer ketamine infusions using a locked infusion pump and patients are maintained on a cardiorespiratory monitor (heart and respiratory rate, blood pressure, and pulse oximeter). Criteria to receive subanesthetic doses of ketamine infusions include inadequate analgesia with standard pain therapy, opioid tolerance associated with undesirable side effects, opioid-induced hyperalgesia, neuropathic pain, postoperative pain in patients in whom opioid use was undesirable, or an opioid-sparing effect was needed for clinical reasons. Ketamine infusions used for end-of-life care were excluded. Exclusion criteria for ketamine administration include history of psychosis, intracranial hypertension, severe labile hypertension, or poorly controlled cardiac arrhythmia, hypersensitivity to ketamine, and age $<3$ months.

In our institution, ketamine infusions order sets are available, and the suggested starting doses are as follows: 1) for opioid naive patients, ketamine is started at $0.05-0.4 \mathrm{mg} /$ $\mathrm{kg} / \mathrm{h}$; 2) for opioid tolerant patients (as documented by the pain service), $0.05-1 \mathrm{mg} / \mathrm{kg} / \mathrm{h}$; and 3) for patients with documented opioid-induced hyperalgesia, $1 \mathrm{mg} / \mathrm{kg} / \mathrm{h}$. During infusions, ketamine doses could be titrated according to its indication and pain scores up to $1 \mathrm{mg} / \mathrm{kg} / \mathrm{h}$ as allowed by the Board of Nursing.

\section{Statistical analysis}

The distributions of raw pain scores, daily opioid intake, and changes over time were tested for normality. Because the data were skewed, the Wilcoxon signed-rank test was used to test for statistical significance of within-individuals changes between baseline and posttreatment outcome measurements.

Box plots were used to examine how the individuallevel changes (between baseline and the day after ketamine discontinuation) in raw pain scores and daily opioid intake were distributed across the subgroups of the eight patient characteristics (age, sex, race, duration of pain, clinical diagnosis, type of pain, location of pain, and duration of ketamine infusion), and the distributions were tested for normality. Because the data were skewed for certain characteristics, nonparametric tests were used for all statistical analyses.

The Kruskal-Wallis test was used to compare the distribution of values across the subgroups of each patient characteristic separately. When an overall difference was detected, Dunn's test (with a Bonferroni adjustment for multiple comparisons) was then used to compare all possible pairs of subgroups and identify any statistically significant detectable differences.

The proportion of patients (95\% confidence interval [CI]) who achieved clinically meaningful reductions in pain and daily opioid intake were graphed in order to visually compare how the proportions varied across the subgroups of all eight patient characteristics.

Fisher's exact test was used to examine whether the proportions varied across the subgroups of sex, race, duration of pain, clinical diagnosis, type of pain, or location of pain. A nonparametric test for trend was used to examine whether the proportion of patients with a clinically meaningful reduction in pain scores and opioid intake systematically declined across ordered age groups or by the duration of ketamine infusion.

Stata 14.1/IC (StataCorp LLC, College Station, TX, USA) was used to conduct all analyses. $p$-values $<0.05$ were considered statistically significant except when Bonferroni's correction for multiple comparisons was applied. In those instances, the statistically significant $p$-value was calculated as $0.05 /$ the total number of comparisons.

\section{Results Study cohort}

We reviewed 1542 records, containing orders for subanesthetic ketamine administration (Figure 1). Records containing ketamine orders not written by the Pain Medicine Division were incomplete or were from patients who had undergone 


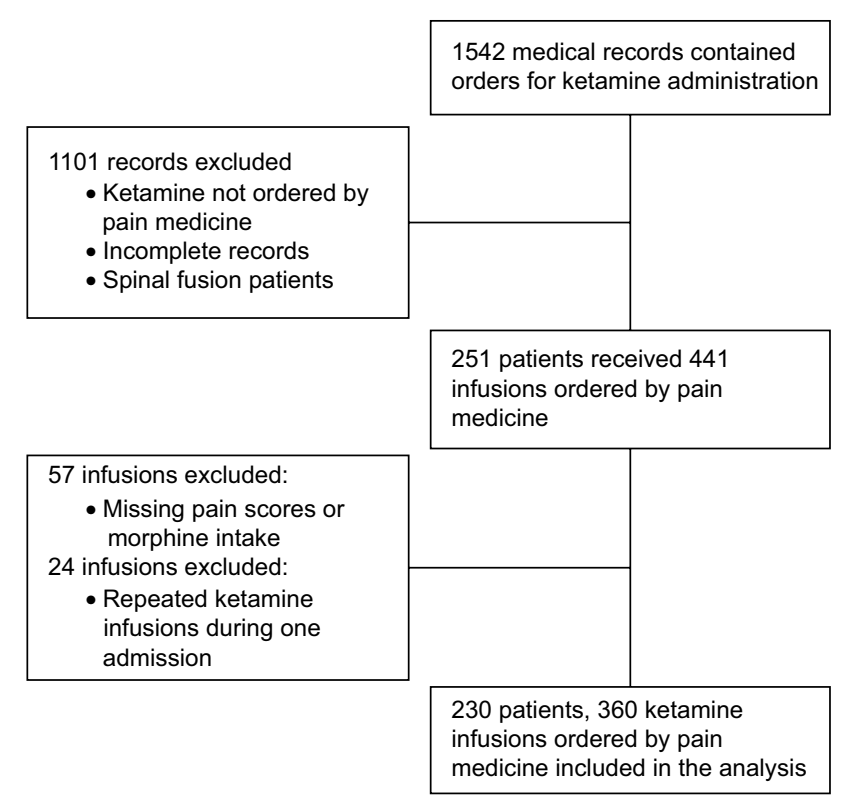

Figure I Flowchart indicating patient selection and exclusion criteria.

spinal fusion (ketamine was administered as part of a postoperative pain management and previously reported ${ }^{11}$ ) were excluded. Two hundred and fifty-one patients and 441 infusions, which occurred between January 2006 and April 2014 , met the study eligibility criteria. Of the 441 remaining records, 57 were excluded as pain scores, and/or opioid intake data were missing during the first or second day of ketamine administration or for the $24 \mathrm{~h}$ after discontinuation of ketamine. Records from 24 ketamine infusions were eliminated because they consisted of a second ketamine infusion during a single hospital admission. The final study cohort included data from 230 different patients who, during 360 separate hospital admissions, received subanesthetic ketamine infusions for pain therapy. Of those, 180 patients were admitted once, while the other 50 were each admitted between two and ten times. For the purpose of this study, each admission was considered an independent event.

\section{Patients}

Patient characteristics at the time of first ketamine infusion are listed in Table 1. The median age was 14 years (interquartile range: $10-17$ years). Over the 360 ketamine infusions, most patients were from 12 to 18 years of age (58\%), 6 to 11 years $(17 \%), 19$ to 21 years $(14 \%), 3$ to 5 years $(5 \%)$, $>22$ years (4\%), and 1.1 to 2.9 years (3\%). Most ketamine infusions lasted 3 days (26\%).

Seventy-eight percent of infusions (280) were administered to patients with acute pain and $22 \%$ to patients with chronic pain. Additionally, most ketamine infusions (50\%)
Table I Characteristics of 230 patients who received 360 ketamine infusions while admitted to a pediatric hospital ${ }^{a}$

\begin{tabular}{ll}
\hline Patients (230) & N (\%) \\
\cline { 1 - 2 } Characteristic & $106(46)$ \\
\cline { 2 - 2 } Sex & $124(54)$ \\
Female & $14(10-17)$ \\
Male & \\
Age (years), median $\left(25^{\text {th }}-75^{\text {th }}\right)$ & $131(57)$ \\
Race/ethnicity & $70(30)$ \\
African-American & $13(6)$ \\
Caucasian & $16(7)$ \\
Hispanic/Latino & \\
Unknown & \\
Ketamine infusions (360) & $203(56)$ \\
Sex & $157(44)$ \\
Female & \\
Male & $229(64)$ \\
Race/ethnicity & $94(26)$ \\
African-American & $15(4)$ \\
Caucasian & $22(6)$ \\
Hispanic/Latino & \\
Unknown & $49(14)$ \\
Duration (days) & $93(26)$ \\
Two & $76(21)$ \\
Three & $52(14)$ \\
Four & $90(25)$ \\
Five & \\
Six or more & \\
\hline Notes: & \\
\hline
\end{tabular}

Notes: apatient's characteristics are those at the time of first ketamine infusion. Race/ethnicity was recorded from a patient self-reported file.

were administered to patients with sickle cell disease who had been admitted for VOE, whereas $17 \%$ who had postoperative pain after surgical trauma and 13\% had malignancy-associated pain (Table 2). Pain locations are listed in Table 3. Most patients had generalized pain (25\%), followed by abdominal (22\%), lower extremity (21\%), and chest (15\%) pain.

\section{Primary outcome}

We collected average pain scores on the day prior to initiation of ketamine and on the first day of ketamine infusion. In the hospital admissions ( $n=331$ of 441 ) where both values were originally available in the electronic medical record, the average pain score on the day prior to ketamine initiation (6.85 [95\% CI: 6.58-7.11]) and on the first day of ketamine infusion were similar (6.84 [95\% CI: 6.59-7.08]), $p=0.499$. Given that in all infusions, the average pain score values were available on the first day of infusion, we considered that as the baseline pain score for the final study cohort of 360 hospital admissions.

Overall, ketamine infusions were associated with significant reductions in average pain scores from the first day of 
Table 2 Clinical diagnosis for patients who received 360 infusions of ketamine in an inpatient setting ${ }^{\mathrm{a}}$

\begin{tabular}{ll}
\hline Associated diagnoses & $\mathbf{n ~ ( \% )}$ \\
\hline Sickle cell disease & $181(50)$ \\
Surgical trauma (postoperative pain) & $61(17)$ \\
Malignancy & $46(13)$ \\
Inflammatory diseases & $18(5)$ \\
Accidental trauma & $16(4)$ \\
Functional gastrointestinal disorder & $14(4)$ \\
Other & $24(7)$ \\
\hline
\end{tabular}

Notes: ${ }^{2}$ Clinical diagnoses were those recorded in the pain medicine consultation. Inflammatory diseases included pancreatitis and Crohn's disease. Other included cystic fibrosis, diabetes mellitus, neurofibromatosis I, postural orthostatic tachycardia syndrome, and two cases simply coded as "other".

Table 3 Pain location in 360 admissions where ketamine was administered to treat pain in a tertiary care pediatric center ${ }^{\mathrm{a}}$

\begin{tabular}{ll}
\hline Pain location & $\mathbf{n}(\%)$ \\
\hline Generalized & $89(25)$ \\
Abdomen & $79(22)$ \\
Lower extremity & $75(21)$ \\
Chest & $53(15)$ \\
Upper extremity & $27(8)$ \\
Back & $25(7)$ \\
Other & $12(3)$ \\
\hline
\end{tabular}

Note: aPain location was that recorded in the pain medicine consultation.

infusion (6.65 [95\% CI: 6.39-6.91]) to the day after discontinuation of ketamine (4.38 [95\% CI: 4.06-4.69]), $p<0.001$.

The effect of ketamine infusions on raw pain scores (within-individual change) was similar comparing all age groups $(p=0.842)$, males and females $(p=0.065)$, all races $(p=0.721)$, acute and chronic pain $(p=0.460)$, and pain types $(p=0.894)$, data not shown. In contrast, the effect of ketamine infusions on pain scores varied according to clinical diagnosis ( $p=0.01)$, infusion duration $(p=0.004)$, and pain location ( $p=0.004$, Figure 2 ). Specifically, greater median reductions in pain scores were observed in patients with malignancy-associated pain and patients with inflammatory disease (pancreatitis and Crohn's disease), whereas the lowest were in patients with functional gastrointestinal disorder and other (cystic fibrosis, diabetes mellitus, neurofribromatosis 1 , postural orthostatic tachycardia syndrome, and two cases simply coded as other, $p=0.011$ for overall difference, Figure 2A). Regarding infusion durations, longer ketamine infusions yielded greater median reductions in pain scores $(p=0.004$, Figure 2B). Further, patients with back $(-2.57$ $[-4.75,-1.56]$, median $\left[25^{\text {th }}, 75^{\text {th }}\right.$ percentiles respectively $]$ ), abdominal $(-1.91[-3.88,-0.6])$, and generalized $(-1.87$ $[-3.43,-0.75])$ pain had the greatest reductions, whereas patients with chest pain $(-0.5[-2.37,-0.33])$ had the lowest reductions in pain scores ( $p=0.004$ for overall difference).

\section{Secondary outcomes}

Regarding opioid intake, ketamine infusions were associated with significant decreases in overall average oral morphineequivalent intake from the first day of infusion $(2.74 \mathrm{mg} / \mathrm{kg} / \mathrm{d}$ [2.47-3.00], mean [95\% CI]) to the day after discontinuation of ketamine $(2.06 \mathrm{mg} / \mathrm{kg} / \mathrm{d}$ [1.81-2.31]), $p<0.001$. There was no impact of age, sex, race, pain duration (acute vs chronic), or pain location or type on the effect of ketamine on oral morphine-equivalent intake (all $p \geq 0.322$ ). However, the ketamine effect on opioid intake varied according to clinical diagnosis $(p=0.030$, Figure $2 \mathrm{C})$ and duration of infusion ( $p=0.022$, Figure 2D). Specifically, greater median reductions in opioid intake were observed in patients with malignancy-associated pain and patients with sickle cell disease, whereas lesser reductions were observed in patients with accidental trauma and postoperative pain after surgical trauma ( $p=0.030$ for overall difference, Figure $2 \mathrm{C}$ ). Regarding ketamine infusion duration, similar to the observation of reduction in pain scores, longer ketamine infusions yielded greater reductions in oral morphine-equivalent intake ( $p=0.022$, Figure 2D).

During ketamine administration, there were no records of hallucinations, changes in sleep pattern, or hemodynamic changes or arrhythmias requiring therapy in any patient.

\section{Clinically meaningful reduction in pain score and morphine-equivalent intake}

We then examined the proportion of ketamine infusions that yielded clinically meaningful changes in pain scores and opioid intake, here defined as a $\geq 20 \%$ reduction in pain scores and $\mathrm{a} \geq 20 \%$ reduction in morphine-equivalent intake after discontinuation of the infusion compared to baseline levels, respectively. Overall, there was a $\geq 20 \%$ reduction in pain scores in $58 \%$ of all ketamine infusions. The proportion of infusions that yielded a $20 \%$ or greater reduction in pain scores varied according to clinical diagnosis ( $p=0.003$ ), pain location $(p=0.012)$, age $(p=0.039)$, and duration of infusion ( $p=0.017$, Figure 3 ). For example, the highest proportion of infusions associated with clinically meaningful reduction in pain scores were observed among infusions administered to cancer patients and patients with inflammatory diseases and the lowest among patients with functional gastrointestinal disorders (Figure 3A). There was a trend for higher frequency of meaningful reduction in pains scores in males (63\%) than in females $(54 \%), p=0.056$. Conversely, the frequency of 
A

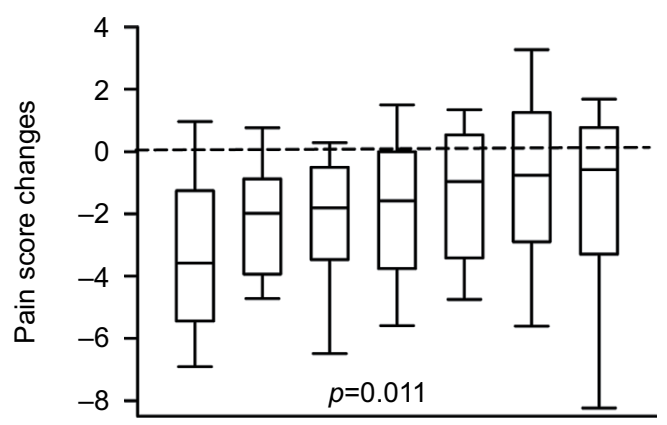

C

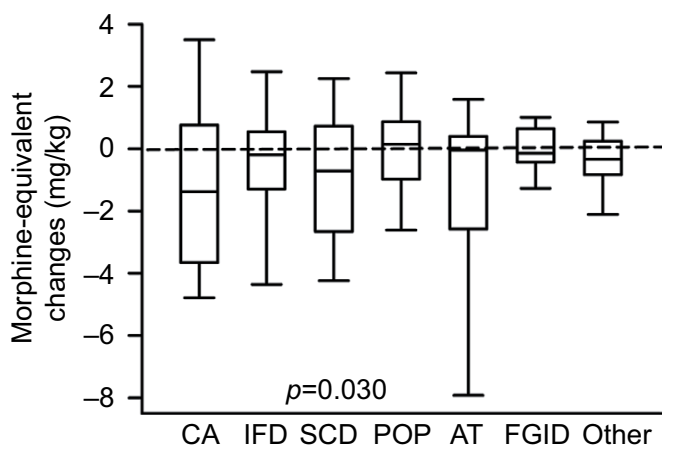

B Infusion duration (days)

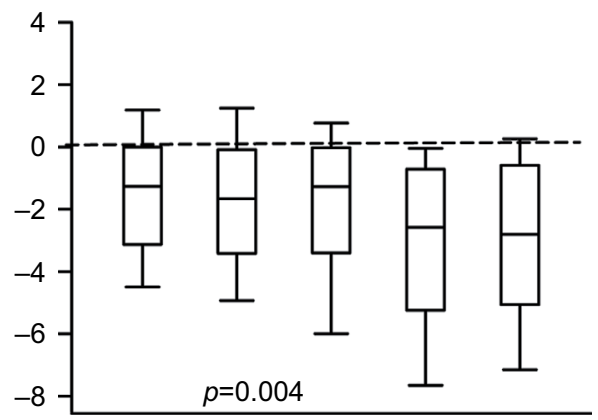

D

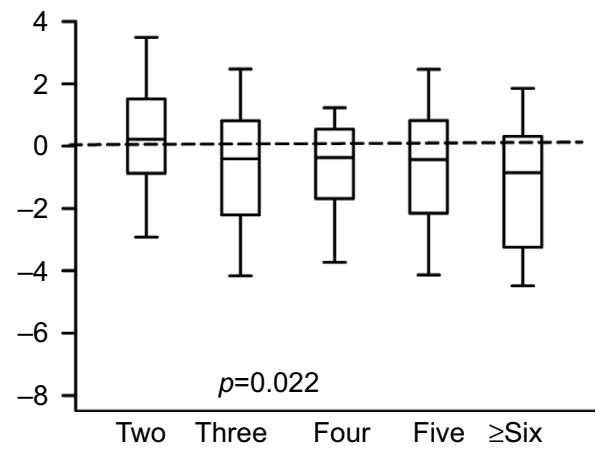

Figure 2 Differential effect of ketamine on pain scores and opioid intake.

Notes: The box plots show median and $25^{\text {th }}$ and $75^{\text {th }}$ percentiles, and the whiskers $10^{\text {th }}$ and $90^{\text {th }}$ percentiles. (A) Box plots of the changes in pain scores from baseline to the day after ketamine discontinuation according to primary clinical diagnosis. Ketamine infusions yielded greater reductions in pain scores in patients with cancer-associated pain and patients with IFD (including pancreatitis and Crohn's disease) and the lowest in patients with FGID and other (cystic fibrosis, diabetes mellitus, neurofibromatosis I, and postural orthostatic tachycardia syndrome), overall difference, $p=0.01 \mathrm{I}$. (B) Effect of ketamine infusions on pain scores also varied according to the duration of ketamine infusion as longer ketamine infusions yielded greater reductions in pain scores $(p=0.004)$. (C) The ketamine effect on opioid intake also varied according to clinical diagnosis $(p=0.030)$ as greater reductions in opioid intake were observed in patients with cancer pain and patients with sickle cell disease, whereas lesser reductions were observed in patients with accidental trauma and postoperative pain due to surgical trauma $(p=0.030)$. (D) Similar to the observation for pain scores, longer ketamine infusions yielded greater reductions in oral morphine-equivalent intake $(p=0.022)$.

Abbreviations: CA, cancer; IFD, inflammatory disease; SCD, sickle cell disease; POP, postoperative pain; AT, accidental trauma; FGID, functional gastrointestinal disease.

meaningful reduction in pain scores did not vary with the type ( $p=0.391)$ and duration of pain $(p=0.224)$ or race $(p=0.268)$.

Regarding the frequency of a meaningful reduction in opioid intake, in $52 \%$ of ketamine infusions there were $20 \%$ or greater reductions in opioid intake. The frequency of infusions associated with $\geq 20 \%$ reduction in opioid intake varied according to duration of ketamine infusion $(p=0.030)$, with longer durations infusions being more likely to result in a meaningful reduction in opioid intake (data not shown). Conversely, the frequency of a meaningful reduction in opioid intake did not vary with the clinical diagnosis, type of pain, duration of pain, location of pain, age, sex, or race.

\section{Discussion}

In this cohort study involving a large number of children, adolescents, and young adults, we examined the effects of subanesthetic ketamine infusions on pain intensity and opioid intake. We found that continuous subanesthetic ketamine infusions administered to children, adolescents, and young adults with inadequate pain control in a regular inpatient care unit setting were safe and not associated with significant psychotropic effects or hemodynamic side effects that warranted treatment. We do acknowledge that increases in blood pressure could occur during administration of ketamine; however, we recorded only instances where changes in blood pressure warranted treatment with vasoactive drugs. Further, while there were no records of hallucinations, it is possible that the presence of hallucinations in young children could have manifested as dysphoria and those were not recorded. Nevertheless, ketamine was associated with significant decreases both in pain scores and opioid intake and these effects varied according to clinical diagnosis, infusion duration, and pain location.

The greatest reductions in pain scores were observed in patients with cancer pain and patients with inflammatory processes including pancreatitis and Crohn's disease, whereas patients with functional gastrointestinal disorder, diabetes mellitus, neurofibromatosis 1 , and postural orthostatic 


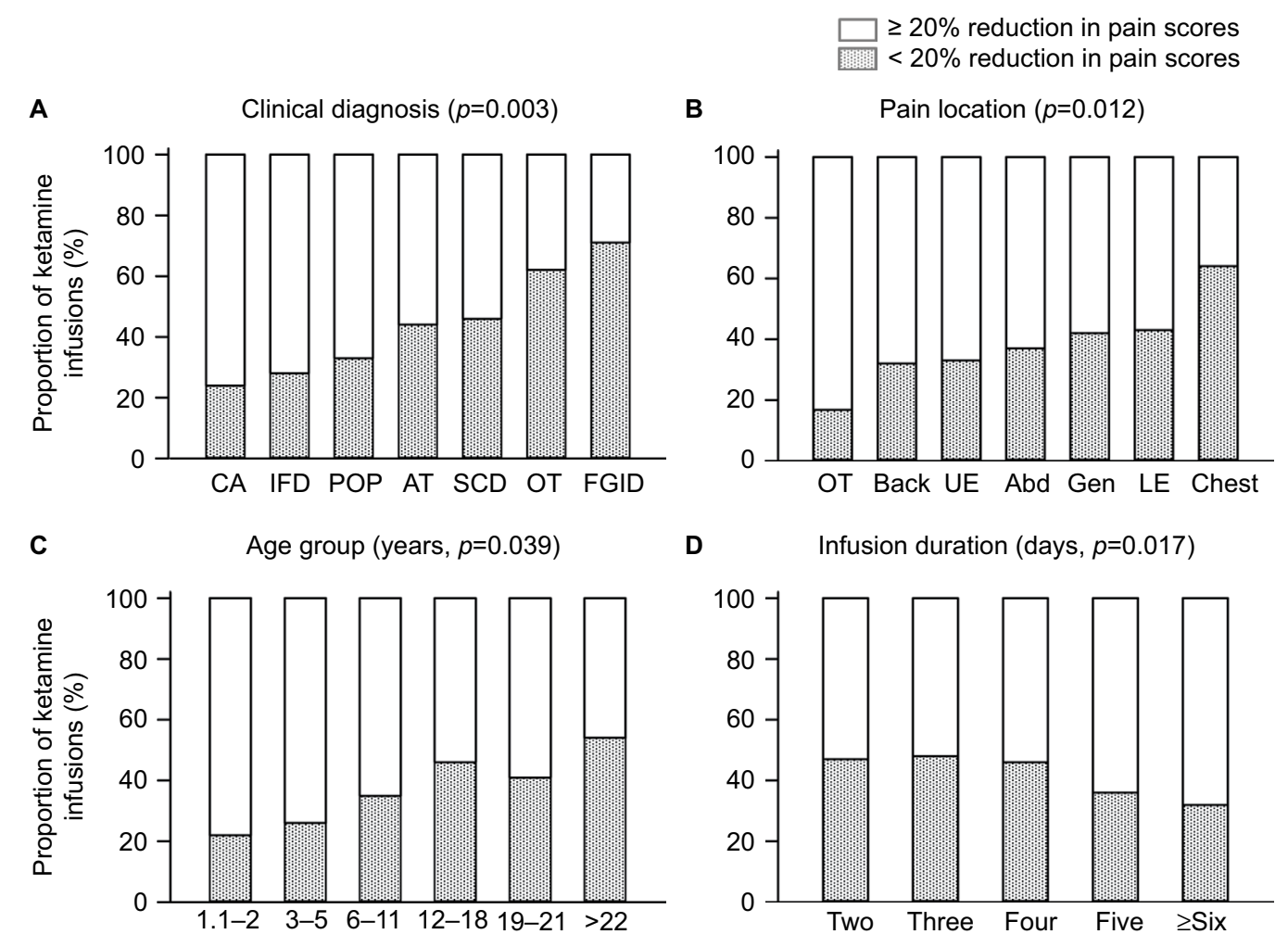

Figure 3 Proportion of ketamine infusions associated with a clinically meaningful reduction in pain scores.

Notes: The stacked bars show the proportion of infusions associated with meaningful ( $\geq 20 \%$ reduction, white bars) and nonmeaningful reductions ( $<20 \%$ reduction, dotted bars). We defined a clinically meaningful reduction as a $\geq 20 \%$ reduction in pain scores from baseline to the day after ketamine discontinuation. The proportion of infusions that yielded a meaningful reduction varied according to clinical diagnosis ( $\mathbf{A}$, overall effect, $p=0.003)$, pain location $(\mathbf{B}, p=0.012)$, age $(\mathbf{C}, p=0.039)$, and duration of infusion (D, $p=0.017$ ).

Abbreviations: CA, cancer; IFD, inflammatory disease; SCD, sickle cell disease; POP, postoperative pain; AT, accidental trauma; OT, other; FGID, functional gastrointestinal disease; UE, upper extremity; Abd, abdominal; Gen, generalized; LE, lower extremity.

tachycardia syndrome had less reduction in pain scores. We also found that ketamine infusions in patients with cancer pain, inflammatory diseases, and postoperative pain were associated with a greater (over $75 \%$ of infusions) frequency of meaningful reduction in pain scores (here defined as a $\geq 20 \%$ reduction). Therefore, this study is informative in that its findings can be used to help design much-needed randomized trials to evaluate the effect of ketamine in the treatment of pain in children, adolescents, and young adults with poorly controlled pain.

Over the past few years, the use of ketamine for the treatment of cancer pain has been the focus of significant debate. ${ }^{15,24,25}$ The discussion ensued after publication of a randomized placebo-controlled clinical trial enrolling 185 adult participants with cancer pain, who received ketamine or placebo delivered subcutaneously. ${ }^{26}$ That trial showed that ketamine, administered over 5 days in a dose-escalating regimen, had no clinical benefit compared to placebo and was associated with increased adverse events. ${ }^{26}$ However, researchers argue that the trial used a subcutaneous regimen not commonly utilized, the ketamine doses were escalated rapidly, and the study population was very heterogeneous, all of which are potential limitations of that trial. ${ }^{25}$ Therefore, researchers argue that those issues should tailor the interpretation of the finding $\mathrm{s}^{26}$ and those negative results should not discard the potential role of ketamine in patients with cancer pain. ${ }^{25}$ After publication of those negative results, a survey of clinicians demonstrated that the practice of using ketamine was continued by $30 \%$ of the respondents ${ }^{24}$ and the use of ketamine to treat cancer pain and other types of pain by many specialists has continued. ${ }^{19,24}$ On balance, one must recognize that the existing data on the use of ketamine in children with cancer pain are very limited, most reports consist of small case series, and randomized clinical trials of ketamine to treat cancer pain in children are lacking. Nevertheless, we have sided with those who argue that, while clinical trials are needed, the practice of using ketamine in children with cancer pain might be one to be adopted when other options are unavailable. 
Concerning acute postoperative pain, we found in the present study that the opioid-sparing effect of ketamine was minimal. This finding is consistent with our results from a previous randomized placebo-controlled trial, where ketamine administered in the intraoperative and postoperative periods did not yield an opioid-sparing effect in adolescents with scoliosis undergoing spinal fusion. ${ }^{11}$ Interestingly, a more recent trial and a new meta-analysis ${ }^{12}$ also showed lack of beneficial effect of ketamine in postoperative pain. ${ }^{27}$ It is important to note that one limitation of the most recent study in adolescents undergoing spinal fusion ${ }^{27}$ as well as ours ${ }^{11}$ is that neither study examined the effect of ketamine on chronic postsurgery pain. Nevertheless, our findings from the present study are discrepant to those showing that low-dose ketamine infusion during the perioperative period yields opioid-sparing effects in adults undergoing major surgeries. ${ }^{28}$ While we acknowledge that, in adults, there are data to support the use of ketamine in the perioperative period, in our institution, we no longer routinely use ketamine to treat postoperative pain in our pediatric patients.

One must acknowledge the limitations of this study, which call for caution in interpreting its results. First, we report on the use of ketamine in a heterogeneous population of children, adolescents, and young adults and lack a control group. Further, while there was consistency regarding the ketamine administration regimen and indications for its use, timing of administration, and titration of opioids and ketamine doses were left to the discretion of the pain medicine attending. Additionally, for the purpose of this study, we regarded ketamine infusions administered to the same patients as independent infusions and we did not account for the potential confounding effect of repeated measures by repeatedly administering ketamine to some patients.

\section{Conclusion}

In conclusion, despite its limitations, this study suggests that in an inpatient setting, the treatment of pain in children, adolescents, and young adults with subanesthetic doses of ketamine is feasible and safe in regular patient care units. The study is also informative in that it suggests that ketamine might have a differential beneficial effect in patients with certain pain syndromes. Additionally, this study raises a number of hypotheses to be tested in future clinical trials to more definitively determine which patients would benefit from this therapeutic modality. Finally, our findings can be used to determine sample and effect sizes for future randomized trials to evaluate the role of ketamine for the treatment of pain in children, adolescents, and young adults.

\section{Acknowledgments}

The authors are grateful to the nursing staff from the Children's National Health System for their excellent care of our patients and their families and to members of the Department of Anesthesia, and Pain Medicine, Critical Care, Acute Care, Pharmacy, the Department of Professional Practice and Nursing Research, the Department of Nursing Staff Development for the development of the low dose ketamine administration program at the Children's National Health System. This study was funded by a grant (RAC 3000193) from the Sheik Zayed Institute for Pediatric Surgical Innovation.

\section{Disclosure}

This article was prepared while Zenaide MN Quezado, MD was employed at the Children's National Health System. The opinions expressed in this article are the author's own and do not reflect the view of the National Institutes of Health, the Department of Health and Human Services, or the United States government. The authors report no other conflicts of interest in this work.

\section{References}

1. Corssen G, Miyasaka M, Domino EF. Changing concepts in pain control during surgery: dissociative anesthesia with CI-581. A progress report. Anesth Analg. 1968;47(6):746-759.

2. Anis NA, Berry SC, Burton NR, Lodge D. The dissociative anaesthetics, ketamine and phencyclidine, selectively reduce excitation of central mammalian neurones by N-methyl-aspartate. Br J Pharmacol. 1983; 79(2):565-575.

3. Mellon RD, Simone AF, Rappaport BA. Use of anesthetic agents in neonates and young children. Anesth Analg. 2007;104(3):509-520.

4. Diazgranados N, Ibrahim L, Brutsche NE, et al. A randomized add-on trial of an N-methyl-D-aspartate antagonist in treatment-resistant bipolar depression. Arch Gen Psychiatry. 2010;67(8):793-802.

5. Finkel JC, Pestieau SR, Quezado ZM. Ketamine as an adjuvant for treatment of cancer pain in children and adolescents. J Pain. 2007;8(6):515-521.

6. Sheehy KA, Muller EA, Lippold C, Nouraie M, Finkel JC, Quezado ZM. Subanesthetic ketamine infusions for the treatment of children and adolescents with chronic pain: a longitudinal study. BMC Pediatr. 2015;15(1):198.

7. Latremoliere A, Woolf CJ. Central sensitization: a generator of pain hypersensitivity by central neural plasticity. J Pain. 2009;10(9):895-926.

8. Trujillo KA. Are NMDA receptors involved in opiate-induced neural and behavioral plasticity? A review of preclinical studies. Psychopharmacology (Berl). 2000;151(2-3):121-141.

9. Angst MS, Koppert W, Pahl I, Clark DJ, Schmelz M. Short-term infusion of the mu-opioid agonist remifentanil in humans causes hyperalgesia during withdrawal. Pain. 2003;106(1-2):49-57.

10. Tawfic QA. A review of the use of ketamine in pain management. J Opioid Manag. 2013;9(5):379-388.

11. Pestieau SR, Finkel JC, Junqueira MM, et al. Prolonged perioperative infusion of low-dose ketamine does not alter opioid use after pediatric scoliosis surgery. Paediatr Anaesth. 2014;24(6):582-590.

12. Michelet D, Hilly J, Skhiri A, et al. Opioid-sparing effect of ketamine in children: a meta-analysis and trial sequential analysis of published studies. Paediatr Drugs. 2016;18(6):421-433.

13. Connolly SB, Prager JP, Harden RN. A systematic review of ketamine for complex regional pain syndrome. Pain Med. 2015;16(5):943-969. 
14. Laskowski K, Stirling A, McKay WP, Lim HJ. A systematic review of intravenous ketamine for postoperative analgesia. Can J Anaesth. 2011;58(10):911-923.

15. Bell RF, Eccleston C, Kalso EA. Ketamine as an adjuvant to opioids for cancer pain. Cochrane Database Syst Rev. 2012;11:Cd003351.

16. Goebel A, Barker C, Turner-Stokes L, et al. Complex Regional Pain Syndrome in Adults: UK Guidelines for Diagnosis, Referral and Management in Primary and Secondary Care. London: Royal College of Physicians; 2012.

17. Perez RS, Zollinger PE, Dijkstra PU, et al. Evidence based guidelines for complex regional pain syndrome type 1. BMC Neurol. 2010;10:20.

18. Butler FK, Kotwal RS, Buckenmaier CC 3rd, et al. A triple-option analgesia plan for tactical combat casualty care: TCCC guidelines change 13-04. J Spec Oper Med. 2014;14(1):13-25.

19. Martinez V, Derivaux B, Beloeil H; Regional Anaesthesia and the Pain Committee of the French Society of Anaesthesiology and Intensive Care. Ketamine for pain management in France, an observational survey. Anaesth Crit Care Pain Med. 2015;34(6):357-361.

20. Lo TC, Yeung ST, Lee S, Skavinski K, Liao S. Reduction of central neuropathic pain with ketamine infusion in a patient with Ehlers-Danlos syndrome: a case report. J Pain Res. 2016;9:683-687.

21. Carr DB, Goudas LC, Denman WT, et al. Safety and efficacy of intranasal ketamine for the treatment of breakthrough pain in patients with chronic pain: a randomized, double-blind, placebo-controlled, crossover study. Pain. 2004;108(1-2):17-27.
22. Mercadante S, Ferrera P, Villari P, Casuccio A, Intravaia G, Mangione S. Frequency, indications, outcomes, and predictive factors of opioid switching in an acute palliative care unit. J Pain Symptom Manage. 2009; 37(4):632-641.

23. Hirschfeld G, Wager J, Schmidt P, Zernikow B. Minimally clinically significant differences for adolescents with chronic pain-variability of ROC-based cut points. J Pain. 2014;15(1):32-39.

24. Hardy JR, Spruyt O, Quinn SJ, Devilee LR, Currow DC. Implementing practice change in chronic cancer pain management: clinician response to a phase III study of ketamine. Intern Med J. 2014;44(6): 586-591.

25. Bell RF, Jaksch W, Kalso EA. Interpreting the evidence: reply to Spruyt et al. J Pain Symptom Manage. 2014;47(4):e2-e4.

26. Hardy J, Quinn S, Fazekas B, et al. Randomized, double-blind, placebocontrolled study to assess the efficacy and toxicity of subcutaneous ketamine in the management of cancer pain. J Clin Oncol. 2012;30(29): 3611-3617.

27. Perello M, Artes D, Pascuets C, Esteban E, Ey AM. Prolonged perioperative low-dose ketamine does not improve short and long-term outcomes after pediatric idiopathic scoliosis surgery. Spine (Phila Pa 1976). 2017;42(5):E304-E312.

28. Guillou N, Tanguy M, Seguin P, Branger B, Campion JP, Malledant Y. The effects of small-dose ketamine on morphine consumption in surgical intensive care unit patients after major abdominal surgery. Anesth Analg. 2003;97(3):843-847.

\section{Journal of Pain Research}

\section{Publish your work in this journal}

The Journal of Pain Research is an international, peer reviewed, open access, online journal that welcomes laboratory and clinical findings in the fields of pain research and the prevention and management of pain. Original research, reviews, symposium reports, hypothesis formation and commentaries are all considered for publication.

\section{Dovepress}

The manuscript management system is completely online and includes a very quick and fair peer-review system, which is all easy to use. Visit http://www.dovepress.com/testimonials.php to read real quotes from published authors. 